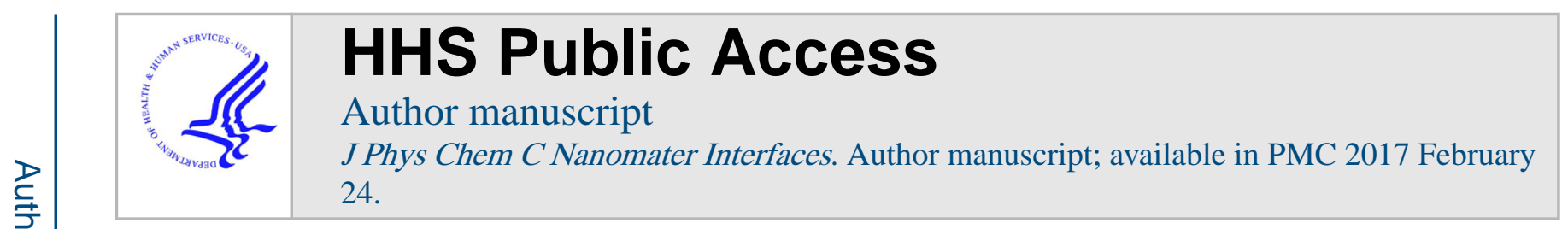

Published in final edited form as:

J Phys Chem C Nanomater Interfaces. 2016 September 22; 120(37): 20886-20895. doi:10.1021/acs.jpcc. $6 \mathrm{~b} 02169$.

\title{
Tuning Localized Surface Plasmon Resonance Wavelengths of Silver Nanoparticles by Mechanical Deformation
}

\author{
Fathima S. Ameer ${ }^{\mathrm{a}}$, Shilpa Varahagiri ${ }^{\mathrm{a}, \mathrm{b}}$, Donald W. Benza ${ }^{\mathrm{a}, \mathrm{c}}$, Daniel R. Willett ${ }^{\mathrm{a}}$, Yimei Wen ${ }^{\mathrm{a}}$, \\ Fenglin Wang ${ }^{\mathrm{a}}$, George Chumanov ${ }^{\mathrm{a}}$, and Jeffrey N. Anker ${ }^{\mathrm{a}, \mathrm{d},{ }^{*}}$ \\ aDepartment of Chemistry, Clemson University, Clemson SC 29634 \\ bDepartment of Mechanical Engineering, Clemson University, Clemson SC 29634 \\ 'Department of Electrical and Computer Engineering, Clemson University, Clemson SC 29634 \\ ${ }^{\mathrm{d} C e n t e r}$ for Optical Materials Science and Engineering Technologies (COMSET), Clemson \\ University, Clemson SC 29634
}

\begin{abstract}
We describe a simple technique to alter the shape of silver nanoparticles (AgNPs) by rolling a glass tube over them to mechanically compress them. The resulting shape change in turn induces a red-shift in the localized surface plasmon resonance (LSPR) scattering spectrum and exposes new surface area. The flattened particles were characterized by optical and electron microscopy, single nanoparticle scattering spectroscopy, and surface enhanced Raman spectroscopy (SERS). AFM and SEM images show that the AgNPs deform into discs; increasing the applied load from 0 to $100 \mathrm{~N}$ increases the AgNP diameter and decreases the height. This deformation caused a dramatic red shift in the nanoparticle scattering spectrum and also generated new surface area to which thiolated molecules could attach as evident from SERS measurements. The simple technique employed here requires no lithographic templates and has potential for rapid, reproducible, inexpensive and scalable tuning of nanoparticle shape, surface area, and resonance while preserving particle volume.
\end{abstract}

\section{TOC Graphic}

\footnotetext{
*Corresponding Author: janker@clemson.edu.

Supporting Information. Characterization of AgNPs, Photograph showing the deformation technique employed, Effect of roll over time on deformation of AgNPs, Effect of rolling speed on deformation of AgNPs, Determination of the contact width of deformation, HRTEM images of single un-deformed and deformed AgNPs, Effect of applied load on different sizes of AgNPs, Surface enhanced Raman spectroscopy measurements, Dark field images of deformed AgNPs in a viscous glycerol/water solution, and angle and polarization-dependent absorption spectra for both un-deformed and deformed AgNPs on glass substrates. This material is available free of charge via the Internet at http://pubs.acs.org.

Author Contributions

The manuscript was written through contributions of all authors. All authors have given approval to the final version of the manuscript. Notes

The authors declare no competing financial interest.
} 


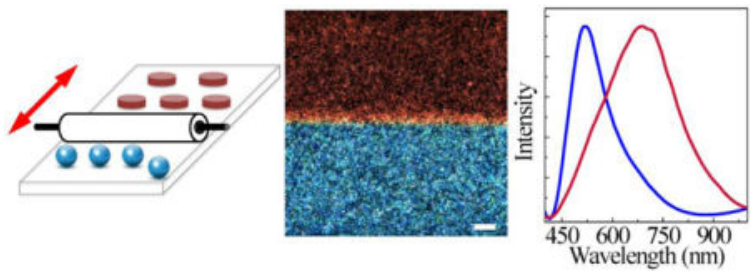

\section{INTRODUCTION}

Noble metal nanoparticles are widely used as optical labels and chemical sensors because they don't photobleach and have large absorption and scattering coefficients at wavelengths which can be tuned by changing the particle size, shape, and refractive index of the surrounding medium. They have been used as labels in immunoassays ${ }^{1-3}$, agents for photothermal therapy ${ }^{4-5}$, lithographic fabrication ${ }^{6-8}$, components in nano-optical switches and waveguides ${ }^{9-10}$, tracers in studying intracellular transport and rotation ${ }^{11}$, in assays to study molecular adsorption kinetics ${ }^{12}$, and in surface enhanced spectroscopies such as: surface enhance Raman spectroscopy (SERS) ${ }^{13-16}$, surface enhanced infrared absorption $(\mathrm{SEIRA})^{17}$, metal enhanced fluorescence $(\mathrm{MEF})^{18}$, and surface enhanced coherent antistokes Raman (SECARS) ${ }^{19}$. Silver nanoparticles (AgNPs) have especially large surface enhancement factors and a localized surface plasmon resonance (LSPR) that can be tuned to any wavelength in the visible and near-infrared range by modifying AgNPs' shape. Methods to control particle shape are therefore important for a wide range of scientific and technological applications.

The shape and size of nanoparticles can be controlled using chemical synthesis techniques $^{20-22}$, nano-lithographic methods ${ }^{23-24}$, and nano indentation ${ }^{25}$. However, each of these techniques produces only one type of particle at a time, and it is difficult to change the shape without affecting volume. Mechanical deformation of the nanoparticles is a rarely employed approach to control shape, discovered by Michael Faraday in 1856. Faraday observed a color change when he ablated a gold film to form gold nanoparticles; this color change reversed after he beat the particles to flatten them back into a film. ${ }^{26-27}$ This work led Faraday to make the (at the time surprising) hypothesis that gold colloids, and similarly colored to the ablated gold, were particulate in nature. His experiments showing color changes in colloids upon aggregation in salt solution and upon drying in gelation (due to decreased inter-particle spacing) supported his hypothesis. Nearly 50 years later, in 1902 Zsigmondy and coworkers confirmed Faraday's hypothesis by observing single gold nanoparticles in dark field microscopy; they also studied changes in the number and color of particles when they aggregated upon salt addition. ${ }^{28}$ More recently, the effects of mechanical strain on the electrical and optical properties of both bulk and nanostructured semiconductors ${ }^{29-30}$ and carbon nanotubes ${ }^{31-32}$ have been reported, often for reversible elastic deformation and occasionally for irreversible plastic deformation. Through computer simulations, Qian and coworkers presented a systematic study of how mechanical strain in the elastic region would affect the LSPR wavelength of metal nanoparticles and enhance the light trapping of metal nanoparticle array of thin film solar cells. ${ }^{33-34}$ Strain has also been applied to correct the dielectric functions of nanoscale metallic nanoparticles. ${ }^{33,}{ }^{35} \mathrm{Li}$ and 
co-authors reported that a $10 \mathrm{~nm}$ silver crystal can be plastically deformed at room temperature in the absence of any dislocation activity by compressing or stretching it using the tip of a scanning tunneling microscope located inside a high-resolution transmission electron microscope, which allows for the deformation of the nanocrystal to be visualized at atomic resolution. ${ }^{36}$ The deformation of nanocrystals has been investigated in $1 \mathrm{D}$ nanowires $^{37}, 2 \mathrm{D}$ nano films ${ }^{38}$, and nanopillars attached to a substrate ${ }^{39}$, by applying pointcontact stress through nanoindentation, however, these single-particle-at-a time experiments have low throughput and have not studied changes in LSPR spectrum. A Master's thesis from our group (2011) showed that gold nanoparticles can be plastically deformed by manually pressing a glass rolling pin over the particles to flatten them, although variation in the force by hand prevented systematic study. ${ }^{40}$ More recently, the strain and morphology evolutions of a $400 \mathrm{~nm}$ sized gold nanocrystal under various high-pressure conditions in a diamond anvil cell were successfully visualized using Bragg CDI measurement. ${ }^{41}$ Huang and co-authors reported the stress-induced deformation process of a single crystal silver nanocube with the edge length about $100 \mathrm{~nm}$, sealed in a panoramic diamond anvil cell. ${ }^{42} \mathrm{In}$ 2015, Bao and co-workers reported tuning the surface plasmon resonance wavelengths of gold nanoparticles by controlling the plastic deformation within a diamond anvil cell. ${ }^{43}$ Although such diamond anvil cells are useful for fundamental studies, they are relatively expensive and have relatively small deformation areas.

Herein we report a simple and straight forward, inexpensive, and scalable way to modify the metal nanoparticle shape by mechanical compression in order to deform nanoparticles and control their LSPR and surface area. ${ }^{44}$ The deformation caused LSPR modulation and surface area variation were characterized by both optical and electron microscopy, single nanoparticle scattering spectroscopy, and SERS.

\section{EXPERIMENTAL SECTION}

\section{Methods and Instrumentation}

All chemicals were obtained from Sigma Aldrich and used as received, except the 2Mercaptobenzimidazole-4,5,6,7- $\mathrm{d}_{4}$ which was acquired from Medical Isotopes, Inc. (Pelham, NH). All aqueous solutions were prepared using deionized (DI) water (18.2 M $\Omega$ $\mathrm{cm})$. Poly(4-vinylpyridine) (PVP) $\left(\mathrm{M}_{\mathrm{w}}=60,000\right)$ was made by dissolving a weighed quantity of PVP into ethanol. Indium-tin oxide (ITO) coated glass $(100 \Omega / \mathrm{cm})$ was purchased from Delta Technologies, Limited. Samples were characterized using enhanced dark field transmission optical microscope (Olympus BX41) equipped with hyperspectral imaging spectrophotometer (CytoViva ${ }^{\circledR}$ Hyperspectral Imaging System (HSI), Auburn, AL). Scanning electron microscopy (SEM) images were collected using a Hitachi SEM-4800 field emission (Hitachi, Japan) electron microscope operating at an accelerating voltage of $15 \mathrm{kV}$ and an average working distance of $8.4 \mathrm{~mm}$. The average nanoparticle size and particle size distribution were obtained using the ImageJ software. High resolution transmission electron microscopy (HRTEM) images were recorded on a Hitachi 9500 microscope operated at $300 \mathrm{kV}$. The surface enhanced Raman spectra (SERS) were recorded with a Leica DMI 5000M microscope equipped with a Delta Nu spectrometer and Andor iDUS420 CCD camera. A 5mW and $632.8 \mathrm{~nm}$ wavelength Helium-Neon laser (REO Inc. 
Boulder, CO, USA) was used as the excitation source for SERS. Alteration of the height of particles was measured using a AIST-NT SmartSPM in non-contact mode with HQ:NSC14/AL BS cantilevers from Mikro-Masch with spring constant of ca. $5.0 \mathrm{~N} / \mathrm{m}$. Absorbance spectra were collected using a Shimadzu UV-2501PC spectrometer.

\section{Poly(4-vinylpyridine) functionalization of indium-tin oxide (ITO) glass substrates}

ITO glass substrates were cleaned by sonication in nanopure water for 15 min followed by drying inside a desiccators and plasma treating for 10 min (Harrick Plasma Cleaner PDC $32 \mathrm{G})$. The cleaned ITO glass substrates were poly(4-vinylpyridine) (PVP) functionalized according to a previously reported method. ${ }^{45}$ After the modification with PVP, substrates were thoroughly rinsed in reagent ethanol to remove all weakly bound polymer molecules leaving only a monolayer directly adhered to the surface. Next, PVP modified substrates were annealed at $120^{\circ} \mathrm{C}$ at ambient atmosphere for a few hours.

\section{AgNP synthesis}

The AgNPs were synthesized using the hydrogen reduction method developed by Chumanov et al. ${ }^{46}$ Briefly, $\mathrm{Ag}_{2} \mathrm{O}$ was added to DI water in a ratio of $1 \mathrm{~g} / \mathrm{L}$ in a quartz reaction vessel. Following this $1 \mathrm{mM}$ sodium silicate was added to promote stabilization of the resulting AgNPs. The mixture was then shaken well before connecting to the condenser and hydrogen line. A heating mantle was placed under the vessel and its contents heated and maintained at $70{ }^{\circ} \mathrm{C}$ with constant stirring. Once the temperature has equilibrated, the vessel is flushed with $\mathrm{H}_{2}$ and pressurized to 10 psi. Hazard: over pressurizing or using a defective vessel may result in explosion and cause severe injuries. Within 10-15 seconds of the initial pressurization, the contents of the vessel become faint yellow in color indicating the formation of metallic silver particles $10-15 \mathrm{~nm}$ in diameter. As the reaction progresses, aliquots can be taken through the spout. Removing the aliquots from the reducing environment stops further growth of the particles. Likewise, the reaction can be stopped at any time simply by releasing the gas from the vessel. In this way, control of the particle diameter is purely a function of total reaction time. The as-synthesized AgNPs with $97 \mathrm{~nm}$ average diameter have a maximum extinction wavelength of $502 \mathrm{~nm}$ and the as-synthesized $\sim$ AgNPs with $64 \mathrm{~nm}$ diameter have a maximum extinction wavelength of $435 \mathrm{~nm}$ (Figure S1, Supporting Information).

\section{Deposition of AgNPs on PVP functionalized ITO glass slides}

To prepare the samples for deformation, both $97 \mathrm{~nm}$ and $64 \mathrm{~nm}$ colloidal AgNPs were diluted with DI water by a factor of 27 to an optical density (O.D.) of 1 (using wavelength at dipole maximum and $1 \mathrm{~cm}$ path length). Previous work involving counting number of particles adsorbed onto a surface indicated that this absorbance corresponds to $\sim 4.3 \times 10^{9}$ particles $/ \mathrm{mL}$ for $97 \mathrm{~nm}$ AgNPs and $\sim 7.4 \times 10^{9}$ particles $/ \mathrm{mL}$ for $64 \mathrm{~nm} \mathrm{AgNPs.}{ }^{46}$ PVP coated glass substrates were immersed in the diluted aqueous suspension of colloidal AgNPs overnight at room temperature to form a monolayer of AgNPs, then rinsed with reagent ethanol and dried at room temperature in a desiccator connected to a vacuum pump overnight. This leads to a well-dispersed configuration of colloidal AgNPs on the substrate surface. 


\section{Mechanical deformation of AgNPs}

A simple rolling pin ( $\mathrm{a}^{1 / 4} \mathrm{~A}^{\prime \prime}$ diameter glass tube with a metal rod through it) was designed to deform AgNPs deposited on PVP functionalized ITO glass slides (Figure 1B). This rolling pin was attached to an ESM 303 with a 1000N Mark 10 Force gauge with computer controllable applied force. AgNPs immobilized on a PVP functionalized ITO glass substrate were then clamped to a linear motorized stage (LTS 300) as shown in the setup photograph Figure S2 (Supporting Information). The rolling pin was placed on one end of the slide to begin with. Mechanical force was applied by the ESM 303 and the stage was moved forward and back to roll the glass tube over the length of the glass substrate 10 times. We see a similar effect with a single roll (Figure S3, Supporting Information). However, single rolled deformed particles appear to have sharper edges compared to the particles deformed by rolling the glass rolling pin 10 times. The applied force was varied from 0 to $100 \mathrm{~N}$ with the number of times the slide was rolled and rolling speed $(500 \mu \mathrm{m} / \mathrm{s})$ were kept consistent for each experiment. Note, bulk glass and silver display minimal mechanical creep, so rolling speed is not expected to affect particle deformation; we saw no significant difference in particle deformation between $100 \mu \mathrm{m} / \mathrm{s}, 500 \mu \mathrm{m} / \mathrm{s}$, and $1000 \mu \mathrm{m} / \mathrm{s}$ rolling rates (Figure S4, Supporting Information). The glass substrates are harder than silver, thus the silver nanoparticles deform instead of the glass; if a material is used with a much lower hardness than silver (e.g., an acrylic roller), the acrylic roller deforms and pulls the AgNPs off the glass slide (data not shown). The glass tubes were cleaned several times using acetone and distilled water prior to use.

\section{Hyperspectral imaging}

Both un-deformed and deformed AgNPs on PVP functionalized ITO glass substrates were examined using enhanced dark field transmission optical microscope (Olympus BX41) equipped with hyperspectral imaging spectrophotometer (CytoViva ${ }^{\circledR}$ Hyperspectral Imaging System (HSI), Auburn, AL). The concentric imaging spectrophotometer was used to record high quality spectrum (high signal-to-noise ratio) in visible and near infrared (VNIR: 400$1000 \mathrm{~nm}$ ) wavelengths at a high spectral resolution of $1.5 \mathrm{~nm}$ with $10 \mathrm{~nm}$ scan size and pixel size $25 \mathrm{~nm}$. The customized hyperspectral imager (mounted on a microscope and controlled by Environment for Visualization software (ENVI 4.4 version) from ITT Visual Solutions) was extracted complete spectral information from single or multiple pixels. The motorized stage was guided by HSI system for synchronizing sample movement with hyperspectral image scanner and the microscope was equipped with a novel illumination system with complete Koehler (fixed) and Critical (adjustable) illumination, which made the nanoparticles appear brighter. All images were acquired using a 40X oil with iris objective at identical gain (gain $=5)$ and exposure time $(0.1 \mathrm{~ms})$.

\section{Extraction of deformed AgNPs in to water and HRTEM acquisition}

Deformed AgNPs were extracted to water by sonication for $1 \mathrm{hr}$. To prepare the grids, a drop of the suspension of either un-deformed or deformed AgNPs were added on carbon-coated Formvar copper grids and then dried at room temperature in a desiccator connected to a vacuum pump overnight. 


\section{Surface enhanced Raman spectral (SERS) acquisition}

The glass substrates used in SERS studies were cleaned in a piranha solution (3:1 conc. $\mathrm{H}_{2} \mathrm{SO}_{4}$ : $30 \% \mathrm{H}_{2} \mathrm{O}_{2}$ ) at $90{ }^{\circ} \mathrm{C}$ for $60 \mathrm{~min}$. (Warning: Piranha solution is hazardous and highly reactive with organic compounds. Extreme care should be taken while handling it). After cooling, the substrates were washed in de-ionized water for $5 \mathrm{~min}$ and stored in a beaker of de-ionized water. Before use, they were plasma etched for 15 minutes (Harrick Plasma Cleaner PDC - 32G). Briefly, $20 \mu \mathrm{L}$ of concentrated colloidal AgNPs solutions were drop coated on a clean glass slide and allowed to dry. This glass slide was incubated in ethanolic solution of $1 \mathrm{mM}$ 2-Mercaptobenzimidazole (MBI- $\mathrm{d}_{0}$ ) overnight, washed with reagent ethanol and allowed to dry before acquiring a SERS spectrum. After SERS spectral acquisition, the un-deformed AgNPs sample was again incubated in MBI- $\mathrm{d}_{0}$ for $1 \mathrm{hr}$ and spectrum was collected to determine whether the sample was saturated or if further incubation would increase the SERS intensity. The sample was then deformed as described in the previous section, and the SERS signal was acquired for the same region. This gave the effect of deformation on intensity. Next, $1 \mathrm{mM}$ 2-Mercaptobenzimidazole-4,5,6,7-d 4 (MBI$\mathrm{d}_{4}$ ) was added and spectrum collected again to observe whether the deformation increased available surface area for MBI- $\mathrm{d}_{4}$ binding. In each case, the glass slide was removed and put back on to the microscope stage. By taking dark field images each time and matching them, it was ensured that the same region was focused in all cases. All the SERS spectra were taken with an Olympus 50X objective (NA $=0.55)$, a spectrograph grating of 300 grooves $/ \mathrm{mm}$, and a laser intensity before entering the sample of $\sim 5 \mathrm{~mW}$. A $632.8 \mathrm{~nm}$ wavelength Helium-Neon laser was used as the excitation source for SERS. The spectral integration time was $20 \mathrm{~s}$. The Raman shift was calibrated using the cyclohexane spectrum as reference.

\section{Atomic force microscopy (AFM)}

AFM images were collected for un-deformed and deformed AgNPs adsorbed on PVP functionalized ITO glass substrates using an AIST-NT SmartSPM in non-contact mode with HQ:NSC14/AL BS cantilevers from Mikro-Masch with spring constant of ca. 5.0 N/m. HQ: NSC14/AL BS cantilevers have a radius of uncoated tip about $8 \mathrm{~nm}$ with $40^{\circ}$ full tip cone. AFM image processing was carried out with AIST-NT SPM Control Software. Polynomial background subtraction and line fit corrections were employed in some AFM images for analysis purpose. Un-deformed and deformed AgNPs sizes were measured based on statistics analysis of $\sim 100$ nanoparticles to achieve average sizes with standard deviation.

\section{Optical imaging}

The scattering properties of un-deformed and deformed AgNPs immobilized in glycerol/ water suspension was observed with a Nikon TE2000 microscope in transmission dark field imaging using a 0.8-0.95 numerical aperture (NA) condenser and a variable aperture $100 \mathrm{X}$ oil immersion objective stopped down to just below 0.8 NA. All parameters of the camera were controlled by Camera Control Pro2 software (Nikon Instruments Inc., Melville, NY, USA). 


\section{RESULTS AND DISCUSSION}

Mechanical deformation was used to alter the LSPR of metallic nanoparticles via changing their shapes. Figure 1A is a schematic of our experimental approach of deforming the nanoparticles from the spherical shape to the 'discs' shape. We deposited AgNPs onto a poly(4-vinylpyridine) (PVP) functionalized ITO glass substrate and mechanically deformed them using a rolling pin, comprising a $1 / 4$ " glass tube with a metal pin through it (Figure 1B). The colloidal AgNPs solution used in this experiment had a mean diameter of $97 \mathrm{~nm}$ (by SEM) and gave a strong extinction peak at around $502 \mathrm{~nm}$ (Figure S1, Supporting Information). The AgNPs immobilized on PVP functionalized ITO glass substrates gave an LSPR peak of $\sim 518 \mathrm{~nm}$ (Figure 1E). This LSPR red-shift is likely due to the change in the refractive index of the surrounding medium of the nanoparticles. When these AgNPs were illuminated with white light they appear as bright blue diffraction-limited points under a dark field microscope (Figure 1C, bottom). After deformation, however, every particle turned red in the dark field image as shown in Figure 1C (top) due to change in particle morphology. The LSPR peak of deformed AgNPs shifted toward red (Figure 1D). From dark field hyperspectral images of deformed (Figure 1F) and un-deformed (Figure 1G) AgNPs we can clearly observe the single nanoparticles. Note, in Figure $1 \mathrm{~F}$ and $1 \mathrm{G}$, we used a dilute concentration of as-synthesized AgNPs solution compared to Figure 1C so that we could clearly distinguish single particles. Representative single nanoparticle scattering spectra of deformed and un-deformed AgNPs were shown in Figure 1H and 1I, respectively. These single nanoparticle scattering spectra correspond to the nanoparticles labeled on Figure 1F and $1 \mathrm{G}$.

The un-deformed and deformed AgNPs were imaged using SEM, as shown in Figure 2A and Figures 2(B-E), respectively. Figures 2(a-e) show the corresponding distribution of nanoparticle average diameters (calculated from the area of each particle) for the undeformed and deformed AgNPs. The SEM images revealed that the AgNPs size and shapes varied as the applied load of deformation changed. According to Figure 2A, as-synthesized AgNPs have a mean diameter of $97 \mathrm{~nm}$ based on the measurements of 100 nanoparticles, and a $23 \%$ of coefficient of variation (CV). Applied loads of $10 \mathrm{~N}, 30 \mathrm{~N}, 50 \mathrm{~N}$, and $100 \mathrm{~N}$ caused the mean AgNP diameter to increase to $132 \mathrm{~nm}$ (CV-25\%), $150 \mathrm{~nm}$ (CV-36\%), 166 $\mathrm{nm}(\mathrm{CV}-26 \%)$, and $173 \mathrm{~nm}(\mathrm{CV}-30 \%)$, respectively. Figure $2 \mathrm{~F}$ shows the correlation between the applied load and average diameters of both un-deformed and deformed AgNPs. Apart from an increase in AgNPs diameter we also observed a change in shape of AgNPs after deformation. Initially AgNPs were approximately spherical in shape (Figure 2A). However, after deformation the nanoparticles became elongated discs. The SEM images also reveal the relative uniformity of the deformed AgNP size. Even after deformation AgNPs are well separated and there is no aggregation (except at higher concentrations where particles begin to touch, data not shown). Comparing SEM images of un-deformed and deformed AgNPs we see similar number of nanoparticles per area after deformation (14 particles/ $\mu \mathrm{m}^{2}$ ), and also observe a similar number in dark field imaging indicating that the particles aren't merging together or being removed by the rolling pin. The LSPR red-shift with increasing particle size is well known from literature. ${ }^{20,47}$ The contact width of deformation if the hollow glass rod is down at one spot instead of rolling was experimentally evaluated as 
a function of the applied load (Figure S5, Supporting Information). We observe a blue/green deformed region in between the un-deformed blue region and deformed red region likely due to the radius of curvature of the glass rod. The measured contact width values were 0.104 $\mathrm{mm}, 0.073 \mathrm{~mm}, 0.065 \mathrm{~mm}$, and $0.054 \mathrm{~mm}$ for $100 \mathrm{~N}, 50 \mathrm{~N}, 30 \mathrm{~N}$, and $10 \mathrm{~N}$ load, respectively. The deformation area is consistent with the expected Hertz contact area for a glass tube with $6 \mathrm{~mm}$ outer diameter, and $4 \mathrm{~mm}$ inner diameter pressing into an infinite glass plate. ${ }^{48}$ Based on these contact width measurements the calculated maximum force per particle varied from $2.3 \mu \mathrm{N}, 1.7 \mu \mathrm{N}, 1.1 \mu \mathrm{N}$, and $0.5 \mu \mathrm{N}$ for applied loads $100 \mathrm{~N}, 50 \mathrm{~N}, 30 \mathrm{~N}$, and $10 \mathrm{~N}$ respectively. The maximum applied pressure during the deformation was larger than the yield strength of bulk silver $55 \mathrm{MPa}^{49}$, therefore the forces we are using are sufficient (the small contact area allows this to be done at small forces), however it is not possible to measure the single particle variation in our setup, which rapidly deforms many particles simultaneously.

A more detailed picture of the shape of the single un-deformed and deformed AgNP was obtained by means of HRTEM (Figure S6, Supporting Information). With the help of highresolution images, the crystal structure of the individual un-deformed and deformed AgNPs could be determined. Unlike the previous studies, which were performed on ITO directly after deformation, it was necessary to remove the particles and place onto a more conductive substrate for HRTEM: The deformed AgNPs on PVP functionalized ITO glass substrate were extracted to water through sonication for $1 \mathrm{hr}$ and re-deposited on copper grids for TEM measurements. Figures S6A and S6C show TEM images of un-deformed and deformed AgNPs, respectively. Figure S6A was obtained using sonicated normal AgNPs and Figure S6C was obtained using sonicated deformed AgNPs. Figure 2D and Figure S6C (Supporting information) were obtained using the same deformed AgNPs sample before and after sonication, respectively. If we compare Figure 2D and Figure S6C (Supporting information) it is clear that shape of deformed AgNPs before and after sonication remain essentially unchanged. Figures S6B and S6D show high-resolution lattice image of undeformed and deformed AgNPs, respectively. Here the atomic planes of the AgNPs are visible. From the diffraction patterns corresponding to the HRTEM images the crystal planes can be correlated with silver. The measured interplanar spacing for all the lattice fringes for un-deformed AgNP is $0.222 \mathrm{~nm}$, which corresponds to the [111] reflection of Ag (Figure S6B). Moreover, deformed AgNP has an interplanar spacing of $0.235 \mathrm{~nm}$ for the lattice fringes, within experimental error of the undeformed particles (Figure S6D). ${ }^{50}$ This data further confirmed that the AgNPs consist of single-crystalline silver plates, before and after deformation. A small area in Figure S6D shows a Moiré pattern, which can be attributed to the interference of two sets of planes. The particles often have a flattened elliptical shape, with the long axis along the [111] crystal axis, which may be due to differences in yield stress along the different crystal axes. ${ }^{51-52}$

AFM was used to measure particle height as well as shape. Figures 3(A-E) show AFM images of un-deformed and deformed AgNPs on ITO glass substrates functionalized with PVP to ensure the binding of AgNPs to the substrate surface and prevent their removal on scanning the sample with the cantilever tip. From the AFM images, it is apparent that after flattening with the rolling pin, the height decreased from an average of $85 \pm 9,49 \pm 5,39$ $\pm 5,32 \pm 2$, and $28 \pm 1 \mathrm{~nm}$ for $0,10,30,50$, and $100 \mathrm{~N}$, respectively, which could be seen in 
Figure 3(A-E). From the AFM and electron microscopy images, it is evident that the mechanical deformation method can be applied to tune the particle shape, which should affect the LSPR wavelength.

We next studied the LSPR properties of the un-deformed and deformed AgNPs by recording the dark field single nanoparticle scattering spectra for un-deformed and deformed AgNPs on the ITO substrate, as shown in Figure 4A (based on the measurements of 100 nanoparticles). The LSPR peak red-shifts and broadens with increasing applied load due to the increasing diameter and elongated shape of AgNPs. An overall LSPR shift of $192 \mathrm{~nm}$ was observed when increased the applied load from $0 \mathrm{~N}$ to $100 \mathrm{~N}$. The LSPR could be shifted further toward red upon additional size increases, which was not in our interest, because if too much pressure is applied, the AgNPs were crushed into small nanoparticles. The data in Figure 4B illustrate the correlation between the maximum LSPR wavelength of un-deformed and deformed AgNPs and applied load. The maximum LSPR wavelength increases steadily for applied loads below $1 \mathrm{~N}$, after which the maximum LSPR wavelength increasing rate become slower above $10 \mathrm{~N}$. Such behavior is likely because the height is most sensitive to small applied forces according to AFM results. In addition, as the particle diameter increases, the incident light is no longer interacts homogeneously with AgNPs and the effects of dynamic depolarization and retardation must be considered. ${ }^{53-57}$

In order to emphasize the generality of this deformation technique, effect of applied load on $\sim 64 \pm 2 \mathrm{~nm}$ AgNPs was also studied. Figure S7 (Supporting Information) shows the average dark field single nanoparticle scattering spectra obtained for $\sim 64 \pm 2 \mathrm{~nm}$ and $\sim 97 \pm 2 \mathrm{~nm}$ AgNPs before and after deformation. Both sizes of particles show a narrow LSPR spectra before deformation. After deformation using a $50 \mathrm{~N}$ load, the LSPR peak of both sizes of nanoparticles red-shifted and broadened due to the plastic deformation of AgNPs.

In addition to altering the nanoparticle scattering spectrum, changing the AgNP shape from a sphere to disc or ellipsoid also increased its surface area. Generation of new surface for molecular binding to the deformed AgNPs was observed by surface enhanced Raman spectroscopy (SERS) measurements. SERS is technique which is used to identify and detect molecules adsorbed to a plasmonic nanoparticle on the basis of their Raman signature. The enhancements in the Raman signal obtained when a molecule adsorbs onto silver nanoparticle surfaces are typically on the order of $10^{5}-10^{8}$, with even higher values for single molecules in hot spots. ${ }^{16,58-59}$ There are three ways to increase the SERS signal: first, increasing the amount of analyte bound to the nanoparticles by either modifying the surface to enhance adsorption, or increasing the total surface area available for adsorption. ${ }^{60}$ Second controlling the nanoparticle shape in order to tune the electromagnetic enhancement arising from the coupling of Raman excitation with the nanoparticle LSPR modes. ${ }^{13}$ Third is to select the analyte, surface, and laser wavelength to maximize the chemical enhancement which results from the formation of charge transfer states between the chemisorbed species and the metal surface. ${ }^{61}$ It is hypothesized that our deformation method affects both the particle surface area and electromagnetic enhancement.

SERS studies were performed on un-deformed and deformed AgNPs on PVP functionalized glass substrates by using 2-Mercaptobenzimidazole (MBI- $d_{0}$ ) and 2- 
Mercaptobenzimidazole-4,5,6,7- $\mathrm{d}_{4}\left(\mathrm{MBI}-d_{4}\right)$ as probe analytes. Figure $6 \mathrm{~A}$ illustrates the experimental scheme of the SERS spectral acquisition. Detailed description of the experimental procedure of SERS spectral acquisition is discussed in the Experimental Section. MBI- $d_{0}$ and MBI- $d_{4}$ were chosen as our probe analytes due to several reasons. First, MBI- $d_{0}$ and MBI- $d_{4}$ are organothiols, which have a very high binding affinity to AgNPs. These organothiols can covalently bind to the surface of the AgNPs. The high binding affinity of MBI- $d_{0}$ and MBI- $d_{4}$ is important to ensure complete passivation of probe molecules to the NP surface. Second, the large spectral differences between MBI- $d_{0}$ and MBI- $d_{4}$ make it easier to identify exposure of new surface area after AgNP deformation. For simplicity, multi component mixtures were referred to as $(\mathrm{A} / \mathrm{B}) t / \mathrm{C}$ in which the components $(\mathrm{A} / \mathrm{B})$ in the inner parentheses are mixed together first before the addition of the component $\mathrm{C}$ with a delay time of $t$ into an (A/B) mixture.

Figures $5 \mathrm{~B}(\mathrm{a})$ and $5 \mathrm{~B}(\mathrm{~b})$ show the SERS spectra of MBI- $d_{0}$ and MBI- $d_{4}$ on colloidal AgNPs, respectively. Although the two molecules differ only in four hydrogen/deuterium substitutions, their SERS spectra are dissimilar because the change in mass changes the vibrational frequency of modes associated with the deuterium. The AgNPs deposited glass substrate was incubated in an ethanolic solution of $1 \mathrm{mM} \mathrm{MBI}-d_{O}$ for overnight to ensure complete ligand adsorption onto the NPs surface. The amount of the MBI- $d_{0}$ mixed with AgNPs is more than the monolayer ligand packing capacity of the AgNPs estimated on the basis of the published MBI packing density on gold NPs. ${ }^{62}$ Complete MBI- $d_{O}$ adsorption is important because it enables us to confirm that there is no free surface area available for further ligand adsorption before deformation of the AgNPs. The glass slide was washed with ethanol to remove unbounded MBI- $d_{0}$ molecules and dried before SERS spectral acquisition. The washing and drying steps were repeated before each SERS spectral acquisition. After complete adsorption of MBI- $d_{0}$ the glass substrate was incubated $1 \mathrm{hr}$ in the MBI- $d_{4}$ solution and the SERS spectrum was recorded (Figure $5 \mathrm{~B}(\mathrm{c})$ ). Since there is no detectable MBI- $d_{4}$ SERS spectral feature further indicates that AgNP surface is completely saturated with MBI- $d_{0}$ during the overnight incubation and there is no place for new MBI- $d_{4}$ adsorption onto AgNPs. Next, the MBI- $d_{0}$ adsorbed AgNP sample was deformed using $50 \mathrm{~N}$ load. The deformation procedure is the same as discussed in the Experimental section. After deformation, there was no detectable change in the intensity of the resulting MBI- $d_{0}$ SERS spectrum (Figure S8(c,d), Supporting Information). Finally, the deformed AgNPs containing glass substrate was incubated in a MBI- $d_{4}$ solution for $1 \mathrm{hr}$ and the SERS spectrum was recorded (Figure $5 \mathrm{~B}(\mathrm{~d})$ ). Apart from the MBI- $d_{0}$ features MBI- $d_{4}$ spectral features could be clearly seen in Figure 5B(d), which we attribute to adsorption of MBI- $d_{4}$ on AgNP due the increased surface area of the particles after deformation. MBI- $d_{4}$ peak intensity increase was $28 \%$ of the MBI- $d_{0}$ peak intensity; for comparison, a 1:1 mixture of MBI- $d_{4}$ :MBI- $d_{0}$ gives a MBI- $\mathrm{d}_{4}$ peak intensity ratio of $97 \% .{ }^{63}$ This signal depends upon both the location and amount of new surface area generated (e.g., the new surface could be in more enhancing regions, especially cracks and edges). It cannot be used to estimate surface area on its own, although it does clearly show that new surface area is available for binding.

Many applications of AgNPs require particles suspended in solution rather than on a flat surface, for example for studying mechanical properties of a medium or intracellular transport using particle tracking. AgNPs are well suited to these applications because they 
provide a bright signal that does not bleach, and rotation can be measured simultaneously with translation because the nanoparticle scattering is orientation dependent. ${ }^{64}$ In addition, drying the particles prior to deformation might be unnecessary if the particles are to be resuspended in solution afterwards. We therefore performed a deformation with a wet colloidal solution of particles, and recollected the solution immediately after deformation to study their Brownian rotational and translational diffusion. The particles were added to glycerol (diluted to $\sim 99 \%$ glycerol) to slow the Brownian diffusion. The particles were then viewed with a linearly polarized filter inserted in the collection optics before the camera. The un-deformed AgNPs strongly scatter blue-green light (the increased refractive index of the glycerol solution causes some red-shift compared to in water). The un-deformed particle scattering intensity did not fluctuate greatly, and there was minimal color change (Figure $6 \mathrm{~A}(1-4))$, likely because the particles had approximately equal axes, although they were not perfectly spherical (Figure S1 and 2A). Note, motion in and out of focus also causes intensity fluctuations, but also causes the particles to blur and form diffraction rings which distinguish this type of motion from rotation-induced blinking relative to the incident light polarization. By contrast, the mechanically deformed AgNPs appeared to blink and change color in the dark field images from blue to red as the polarized light excites the transverse or longitudinal axes (Figures 6B(1-4)). By taking a series of images in time, we can observe changes in orientation and infer rotational velocity, which provides useful information related to the physical and chemical surroundings. ${ }^{65}$ Although the deformation process was performed directly in solution, the process can also be performed after drying particles on a dry substrate and transferring deformed AgNPs to solution as shown in Figure S9

(Supporting Information). This orientation-dependent color change qualitatively agrees with extinction measurements for particles on a glass slide as the slide is angled with respect to the incident UV-vis light beam (Figure S10). For flattened AgNP, a transverse mode in the $350-400 \mathrm{~nm}$ spectral region (blue light) appears as the angle increases for s-polarized light (polarization axis along the particle height at large incident angles), see Figure S10C; for ppolarized light (polarization axis along the particle diameter at all incident angles), similar spectra are observed at all angles, see Figure S10D. By contrast, there is no significant orientation-dependencies in the extinction peak shape for un-deformed particles under either s- or p-polarized light (see Figures S10A and S10B), which was expected because the undeformed particles are approximately spherical with equal height and diameter.

\section{CONCLUSIONS}

In summary, we present a simple, inexpensive yet effective technique to deform plasmonic AgNPs by mechanically rolling a glass tube over NPs while maintaining particle volume. LSPR wavelengths can be effectively controlled through deformation which is evident from the microscopy and spectroscopy results. This method does not require any chemical reagents, molds or templates and requires very little time to perform. Future works involve using the NPs to track rotation during intra cellular transport, as well as more fundamental studies of how deformation and patterning affects LSPR and SERS. We will also apply this methodology to deforming copper NPs, magnetic iron, cobalt and nickel NPs. The simplicity, versatility, and speed of this method would be advantageous for larger-scale 
production of non-spherical plasmonic NPs. While demonstrated here for AgNPs, this new approach can be extended to other systems.

\section{Supplementary Material}

Refer to Web version on PubMed Central for supplementary material.

\section{Acknowledgments}

The material is based upon work supported by NIBIB of the National Institutes of Health under award number R15EB014560-01A1 and NIGMS of the National Institutes of Health under award number 5P20GM103444-07.

\section{References}

1. Schultz S, Smith DR, Mock JJ, Schultz DA. Single-Target Molecule Detection with Nonbleaching Multicolor Optical Immunolabels. Proc Natl Acad Sci USA. 2000; 97:996-1001. [PubMed: 10655473]

2. Yguerabide J, Yguerabide EE. Light-Scattering Submicroscopic Particles as Highly Fluorescent Analogs and Their Use as Tracer Labels in Clinical and Biological Applications: I. Theory. Anal Biochem. 1998; 262:137-156. [PubMed: 9750128]

3. Nam J-M, Thaxton CS, Mirkin CA. Nanoparticle-Based Bio-Bar Codes for the Ultrasensitive Detection of Proteins. Science. 2003; 301:1884-1886. [PubMed: 14512622]

4. Jaque D, Martinez Maestro L, del Rosal B, Haro-Gonzalez P, Benayas A, Plaza JL, Martin Rodriguez E, Garcia Sole J. Nanoparticles for Photothermal Therapies. Nanoscale. 2014; 6:94949530. [PubMed: 25030381]

5. Rosi NL, Giljohann DA, Thaxton CS, Lytton-Jean AKR, Han MS, Mirkin CA. OligonucleotideModified Gold Nanoparticles for Intracellular Gene Regulation. Science. 2006; 312:1027-1030. [PubMed: 16709779]

6. Srituravanich W, Fang N, Sun C, Luo Q, Zhang X. Plasmonic Nanolithography. Nano Lett. 2004; 4:1085-1088.

7. Kik PG, Maier SA, Atwater HA. Surface Plasmons for Nanofabrication. 2004:215-223.

8. Sundaramurthy A, Schuck PJ, Conley NR, Fromm DP, Kino GS, Moerner WE. Toward NanometerScale Optical Photolithography: Utilizing the Near-Field of Bowtie Optical Nanoantennas. Nano Lett. 2006; 6:355-360. [PubMed: 16522022]

9. Mulvaney P, Pérez-Juste J, Giersig M, Liz-Marzán L, Pecharromán C. Drastic Surface Plasmon Mode Shifts in Gold Nanorods Due to Electron Charging. Plasmonics. 2006; 1:61-66.

10. Boltasseva A, Shalaev VM. All That Glitters Need Not be Gold. Science. 2015; 347:1308-1310. [PubMed: 25792313]

11. Nguyen KVT, Anker JN. Detecting De-gelation through Tissue Using Magnetically Modulated Optical Nanoprobes (MagMOONs). Sensor Actuat B-Chem. 2014; 205:313-321.

12. Niemeyer CM. Nanoparticles, Proteins, and Nucleic Acids: Biotechnology Meets Materials Science. Angew Chem Int Edit. 2001; 40:4128-4158.

13. Jeanmaire DL, Van Duyne RP. Surface Raman Spectroelectrochemistry: Part I. Heterocyclic, Aromatic, and Aliphatic Amines Adsorbed on the Anodized Silver Electrode. J Electroanal Chem Interfacial Electrochem. 1977; 84:1-20.

14. Nie S, Emory SR. Probing Single Molecules and Single Nanoparticles by Surface-Enhanced Raman Scattering. Science. 1997; 275:1102-1106. [PubMed: 9027306]

15. Willets KA, Duyne RPV. Localized Surface Plasmon Resonance Spectroscopy and Sensing. Annu Rev Phys Chem. 2007; 58:267-297. [PubMed: 17067281]

16. Anker JN, Hall WP, Lyandres O, Shah NC, Zhao J, Van Duyne RP. Biosensing with Plasmonic Nanosensors. Nat Mater. 2008; 7:442-453. [PubMed: 18497851]

17. Zhang, S. Introduction to Modern Raman Spectroscopy II-New Raman Spectroscopic Branch Classified Based on Applied Objects. John Wiley \& Sons, Inc.; U.S.A.: 2012. 
18. Zhang J, Fu Y, Chowdhury MH, Lakowicz JR. Metal-Enhanced Single-Molecule Fluorescence on Silver Particle Monomer and Dimer: Coupling Effect between Metal Particles. Nano Lett. 2007; 7:2101-2107. [PubMed: 17580926]

19. Zhang Y, Zhen YR, Neumann O, Day JK, Nordlander P, Halas NJ. Coherent Anti-Stokes Raman Scattering with Single-Molecule Sensitivity using a Plasmonic Fano Resonance. Nat Commun. 2014; 5:1-7.

20. Sun Y, Xia Y. Shape-Controlled Synthesis of Gold and Silver Nanoparticles. Science. 2002; 298:2176-2179. [PubMed: 12481134]

21. Nikoobakht B, El-Sayed MA. Preparation and Growth Mechanism of Gold Nanorods (NRs) Using Seed-Mediated Growth Method. Chem Mater. 2003; 15:1957-1962.

22. Yang Z, Qian H, Chen H, Anker JN. One-Pot Hydrothermal Synthesis of Silver Nanowires via Citrate Reduction. J Colloid Interface Sci. 2010; 352:285-291. [PubMed: 20869063]

23. Tan H, Gilbertson A, Chou SY. Roller Nanoimprint Lithography. J Vac Sci Technol B. 1998; 16:3926-3928.

24. Canelas DA, Herlihy KP, DeSimone JM. Top-down particle fabrication: control of size and shape for diagnostic imaging and drug delivery. Wiley Interdiscip Rev Nanomed Nanobiotechnol. 2009; 1:391-404. [PubMed: 20049805]

25. Zhang ZL, Kristiansen H, Liu J. A Method for Determining Elastic Properties of Micron-Sized Polymer Particles by Using Flat Punch Test. Comput Mater Sci. 2007; 39:305-314.

26. Faraday M. The Bakerian Lecture: Experimental Relations of Gold (and Other Metals) to Light. Philos Trans R Soc Lond. 1857; 147:145-181.

27. Tweney RD. Discovering Discovery: How Faraday Found the First Metallic Colloid. Perspect Sci. 2006; 14:97-121.

28. Siedentopf H, Zsigmondy R. Uber Sichtbarmachung und Größenbestimmung ultramikoskopischer Teilchen, mit besonderer Anwendung auf Goldrubingläser. Ann Phys. 1902; 315:1-39.

29. Pistol ME, Carlsson N, Persson C, Seifert W, Samuelson L. Observation of Strain Effects in Semiconductor Dots Depending on Cap Layer Thickness. Appl Phys Lett. 1995; 67:1438-1440.

30. Audoit G, Mhuircheartaigh EN, Lipson SM, Morris MA, Blau WJ, Holmes JD. Strain Induced Photoluminescence from Silicon and Germanium Nanowire Arrays. J Mater Chem. 2005; 15:4809-4815.

31. Liu B, Jiang H, Johnson HT, Huang Y. The Influence of Mechanical Deformation on the Electrical Properties of Single Wall Carbon Nanotubes. J Mech Phys Solids. 2004; 52:1-26.

32. Heyd R, Charlier A, McRae E. Uniaxial-stress effects on the electronic properties of carbon nanotubes. Phys Rev B. 1997; 55:6820-6824.

33. Qian X, Park HS. The Influence of Mechanical Strain on the Optical Properties of Spherical Gold Nanoparticles. J Mechan Phys Solids. 2010; 58:330-345.

34. Xiaohu, Q., Jing, B. Strain-Tuned Light Trapping Performance of Plasmonic Solar Cells. Photovoltaic Specialists Conference (PVSC), 2011 37th IEEE; 19-24 June 2011; 2011. p. 000669-000672.

35. Xiaohu Q, Harold SP. Strain Effects on the SERS Enhancements for Spherical Silver Nanoparticles. Nanotechnology. 2010; 21:365704. [PubMed: 20699483]

36. Sun J, He L, Lo Y-C, Xu T, Bi H, Sun L, Zhang Z, Mao SX, Li J. Liquid-Like Pseudoelasticity of Sub-10-nm Crystalline Silver Particles. Nat Mater. 2014; 13:1007-1012. [PubMed: 25306422]

37. Wang L, Liu P, Guan P, Yang M, Sun J, Cheng Y, Hirata A, Zhang Z, Ma E, Chen M. In situ Atomic-Scale Observation of Continuous and Reversible Lattice Deformation Beyond the Elastic Limit. Nat Commun. 2013; 4:1-7.

38. Chen M, Ma E, Hemker KJ, Sheng H, Wang Y, Cheng X. Deformation Twinning in Nanocrystalline Aluminum. Science. 2003; 300:1275-1277. [PubMed: 12714676]

39. Yu Q, Shan Z-W, Li J, Huang X, Xiao L, Sun J, Ma E. Strong Crystal Size Effect on Deformation Twinning. Nature. 2010; 463:335-338. [PubMed: 20090749]

40. Varahagiri, VS. Masters. Clemson University; 2011. Fabrication and Characterization of MultiFunctional Non-Spherical Particles via Mechanical Deformation. 
41. Yang W, Huang X, Harder R, Clark JN, Robinson IK, Mao H-k. Coherent Diffraction Imaging of Nanoscale Strain Evolution in a Single Crystal Under High Pressure. Nat Commun. 2013; 4:1680. [PubMed: 23575684]

42. Huang X, Yang W, Harder R, Sun Y, Lu M, Chu YS, Robinson IK, Mao H-k. Deformation Twinning of a Silver Nanocrystal under High Pressure. Nano Lett. 2015; 15:7644-7649. [PubMed: 26484941]

43. Bao Y, Zhao B, Tang X, Hou D, Cai J, Tang S, Liu J, Wang F, Cui T. Tuning Surface Plasmon Resonance by the Plastic Deformation of Au Nanoparticles within a Diamond Anvil Cell. Appl Phys Lett. 2015; 107:201909.

44. Anker JN, Horvath TD, Kopelman R. Cooking With Nanoparticles: A Simple Method of Forming Roll, Pancake and Breaded Polystyrene Microparticles. Eur Cell Mater. 2002; 3:95-97.

45. Malynych S, Luzinov I, Chumanov G. Poly(Vinyl Pyridine) as a Universal Surface Modifier for Immobilization of Nanoparticles. J Phys Chem B. 2002; 106:1280-1285.

46. Evanoff DD, Chumanov G. Size-Controlled Synthesis of Nanoparticles. 2. Measurement of Extinction, Scattering, and Absorption Cross Sections. J Phys Chem B. 2004; 108:13957-13962.

47. Zheng YB, Juluri BK, Mao X, Walker TR, Huang TJ. Systematic Investigation of Localized Surface Plasmon Resonance of Long-Range Ordered Au Nanodisk Arrays. J Appl Phys. 2008; 103:014308.

48. Darji, P., Vakharia, D. Development of Graphical Solution to Determine Optimum Hollowness of Hollow Cylindrical Roller Bearing Using Elastic Finite Element Analysis. InTech; U.S.A.: 2012.

49. Zhu Y, Qin Q, Xu F, Fan F, Ding Y, Zhang T, Wiley BJ, Wang ZL. Size Effects on Elasticity, Yielding, and Fracture of Silver Nanowires: In situ experiments. Phys Rev B. 2012; 85:045443.

50. Aiwei T, Yu W, Haihang Y, Chao Z, Chunhe Y, Xu L, Hongshang P, Fujun Z, Yanbing H, Feng T. Controllable Synthesis of Silver and Silver Sulfide Nanocrystals via Selective Cleavage of Chemical Bonds. Nanotechnology. 2013; 24:355602. [PubMed: 23924847]

51. Koski KJ, Kamp NM, Smith RK, Kunz M, Knight JK, Alivisatos AP. Structural Distortions in 5$10 \mathrm{~nm}$ Silver Nanoparticles under High Pressure. Phys Rev B. 2008; 78:165410.

52. Li B, Wen X, Li R, Wang Z, Clem PG, Fan H. Stress-Induced Phase Transformation and Optical Coupling of Silver Nanoparticle Superlattices Into Mechanically Stable Nanowires. Nat Commun. 2014; 5:1-7.

53. Kelly KL, Coronado E, Zhao LL, Schatz GC. The Optical Properties of Metal Nanoparticles: The Influence of Size, Shape, and Dielectric Environment. J Phys Chem B. 2002; 107:668-677.

54. Myroshnychenko V, Rodriguez-Fernandez J, Pastoriza-Santos I, Funston AM, Novo C, Mulvaney P, Liz-Marzan LM, Garcia de Abajo FJ. Modelling the Optical Response of Gold Nanoparticles. Chem Soc Rev. 2008; 37:1792-1805. [PubMed: 18762829]

55. Wokaun A, Gordon JP, Liao PF. Radiation Damping in Surface-Enhanced Raman Scattering. Phys Rev Lett. 1982; 48:957-960.

56. Meier M, Wokaun A. Enhanced Fields on Large Metal Particles: Dynamic Depolarization. Opt Lett. 1983; 8:581-583. [PubMed: 19718190]

57. Noguez C. Optical Properties of Isolated and Supported Metal Nanoparticles. Opt Mater. 2005; 27:1204-1211.

58. Haes AJ, Haynes CL, McFarland AD, Schatz GC, Van Duyne RP, Zou S. Plasmonic materials for surface-enhanced sensing and spectroscopy. MRS Bulletin. 2005; 30:368-375.

59. Ameer FS, Hu W, Ansar SM, Siriwardana K, Collier WE, Zou S, Zhang D. Robust and Reproducible Quantification of SERS Enhancement Factors Using a Combination of TimeResolved Raman Spectroscopy and Solvent Internal Reference Method. J Phys Chem C. 2013; 117:3483-3488.

60. Fleischmann M, Hendra PJ, McQuillan AJ. Raman Spectra of Pyridine Adsorbed at a Silver Electrode. Chem Phys Lett. 1974; 26:163-166.

61. Albrecht MG, Creighton JA. Anomalously Intense Raman Spectra of Pyridine at a Silver Electrode. J Am Chem Soc. 1977; 99:5215-5217.

62. Ansar SM, Haputhanthri R, Edmonds B, Liu D, Yu L, Sygula A, Zhang D. Determination of the Binding Affinity, Packing, and Conformation of Thiolate and Thione Ligands on Gold Nanoparticles. J Phys Chem C. 2011; 115:653-660. 
63. Zhang D, Ansar SM. Ratiometric Surface Enhanced Raman Quantification of Ligand Adsorption onto a Gold Nanoparticle. Anal Chem. 2010; 82:5910-5914. [PubMed: 20536157]

64. Chan Y, Xu H, Cao L, Tang T, Li D, Sun X. ZnO/Si Arrays Decorated by Au Nanoparticles for Surface-Enhanced Raman Scattering Study. J Appl Phys. 2012; 111:033104-033106.

65. McNaughton BH, Kehbein KA, Anker JN, Kopelman R. Sudden Breakdown in Linear Response of a Rotationally Driven Magnetic Microparticle and Application to Physical and Chemical Microsensing. J Phys Chem B. 2006; 110:18958-18964. [PubMed: 16986890] 


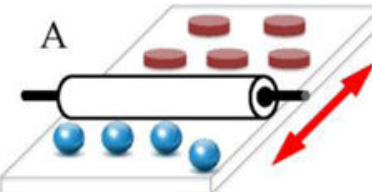

B

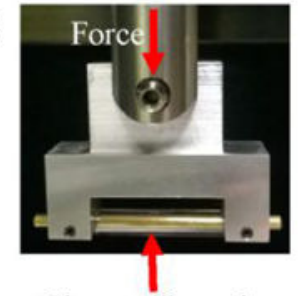

Glass rolling pin

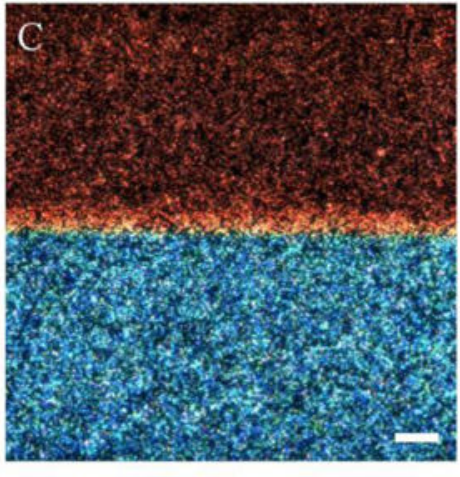

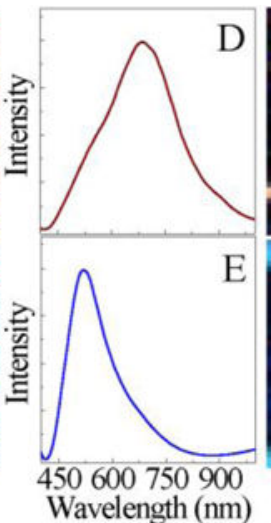
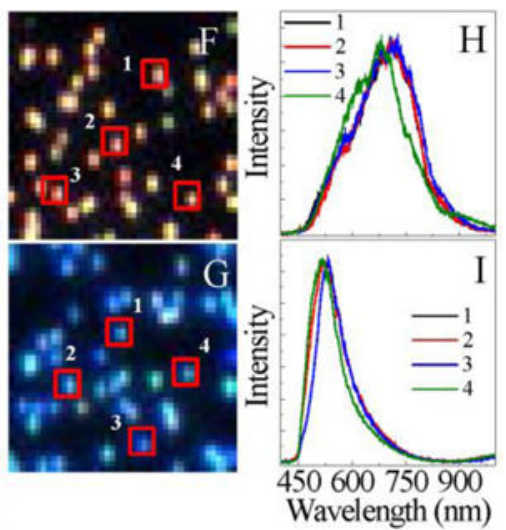

Figure 1.

(A) Schematic of the experimental approach to deform the AgNPs from the initial spherical shape to the final 'discs' shape. AgNPs were deposited onto a PVP functionalized ITO glass substrate. (B) A rolling pin, comprising a $1 / 4$ " glass tube with a metal pin through it, is rolled over particles deposited on a PVP functionalized ITO glass substrate. (C) Dark field microscopy image representing both un-deformed (bottom) and deformed AgNPs (top) on a PVP functionalized ITO glass substrate. Average dark field single nanoparticle scattering spectra of (D) deformed and (E) un-deformed AgNPs.4X zoom in images of (F) deformed and $(\mathrm{G})$ un-deformed AgNPs. Representative dark field single nanoparticle scattering spectra of $(\mathrm{H})$ deformed and (I) un-deformed AgNPs. The squared AgNPs in Figures (F) and (G) produced the signals for $(\mathrm{H})$ and $(\mathrm{I})$, respectively. The spectra are scaled to the same maximum intensity. The different colors are due to the different nanoparticle shapes and sizes. The scale bar of the dark field image is $20 \mu \mathrm{m}$. 

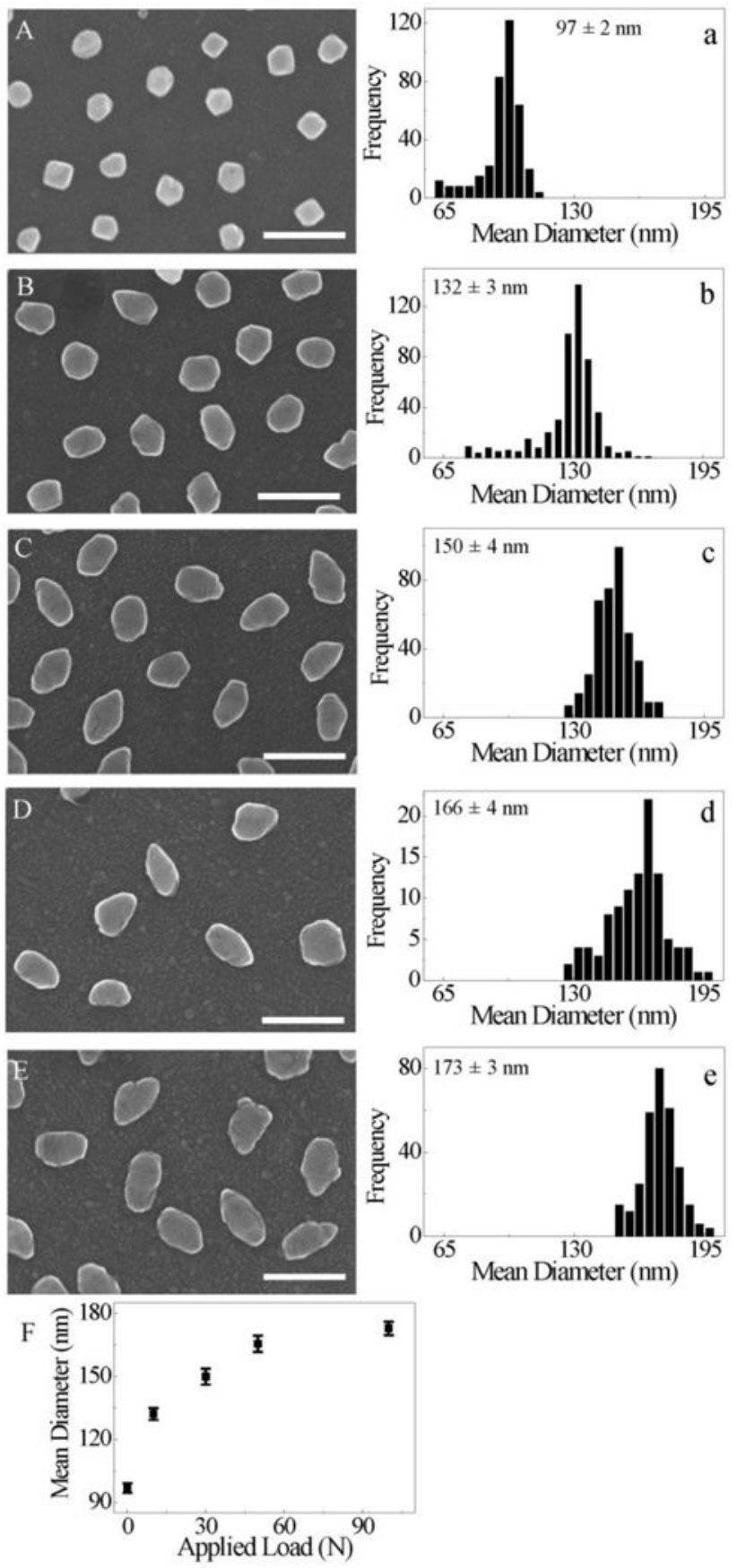

Figure 2.

(Left) SEM images showing effect of applied load (A) $0 \mathrm{~N}$, (B) $10 \mathrm{~N}$, (C) $30 \mathrm{~N}$, (D) $50 \mathrm{~N}$, and (E) $100 \mathrm{~N}$ on deformation of AgNPs. The scale bar of each image is $300 \mathrm{~nm}$. All images have the same magnification. (Right) Histograms of the average particle diameter for (a) undeformed and (b-e) deformed AgNPs. The sample preparation and SEM imaging procedure are described in the Experimental Section. The inset numbers show estimated mean diameter and estimate uncertainty from a Gaussian fit. (F) Increase in mean AgNPs diameter as a function of applied load. 

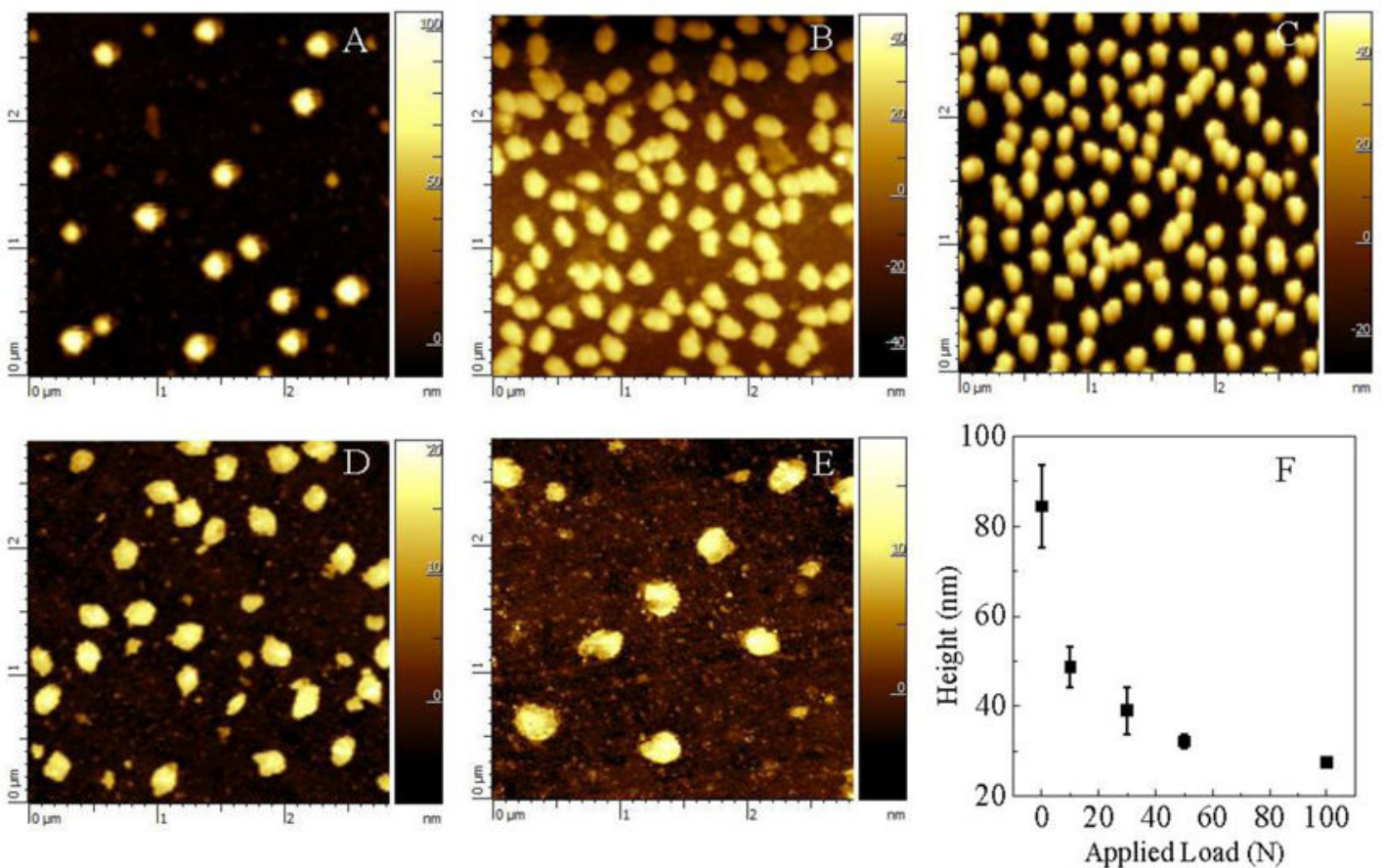

Figure 3.

AFM images showing effect of applied load on deformation of AgNPs. Contact AFM was used to map the profiles of AgNPs on PVP functionalized ITO glass substrates before (A) and after deformation (B-E) by applying (A) $0 \mathrm{~N}$, (B) $10 \mathrm{~N}$, (C) $30 \mathrm{~N}$, (D) $50 \mathrm{~N}$, and (E) 100 $\mathrm{N}$ loads, respectively. (F) Correlation between AgNPs heights estimated from AFM images as a function of applied load. The sample preparation and AFM imaging procedure were described in the Experimental Section. 

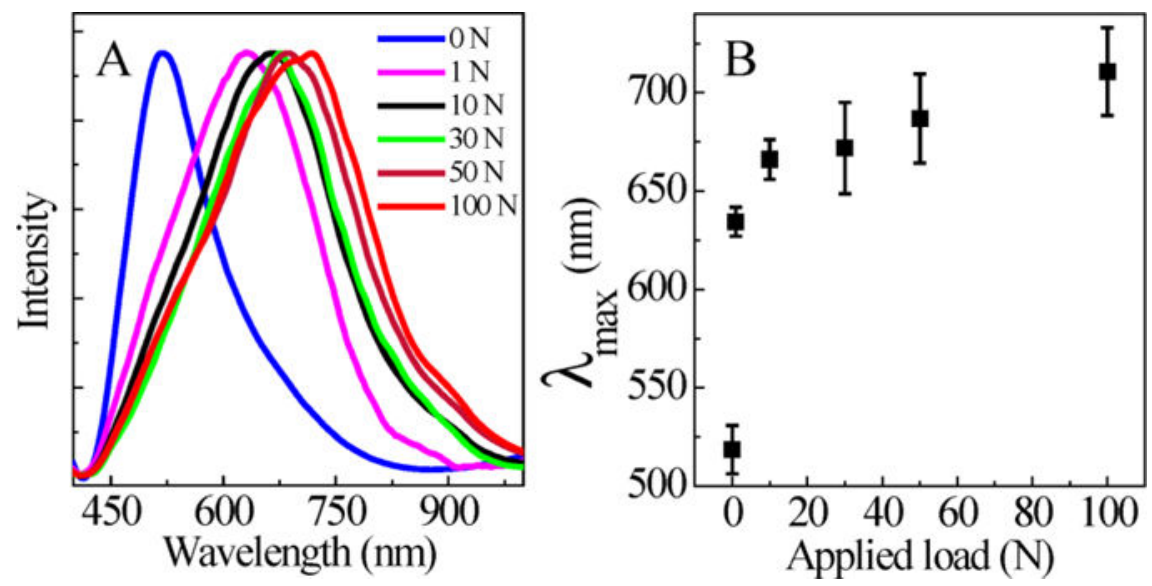

Figure 4.

(A) Average dark field single nanoparticle scattering spectra of un-deformed and deformed AgNPs. The spectra are scaled to the same maximum intensity. (B) Experimental data on the evolution of LSPR peak position, before and after deformation, as a function of applied load. 
A

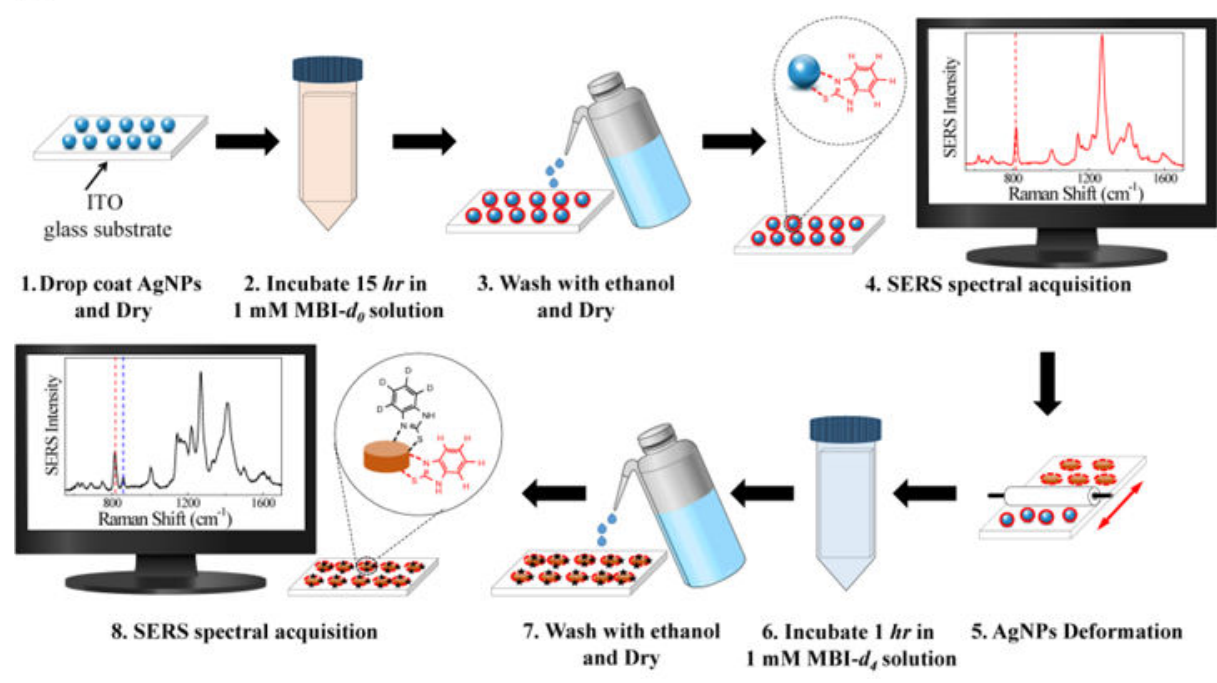

$\mathrm{B}$

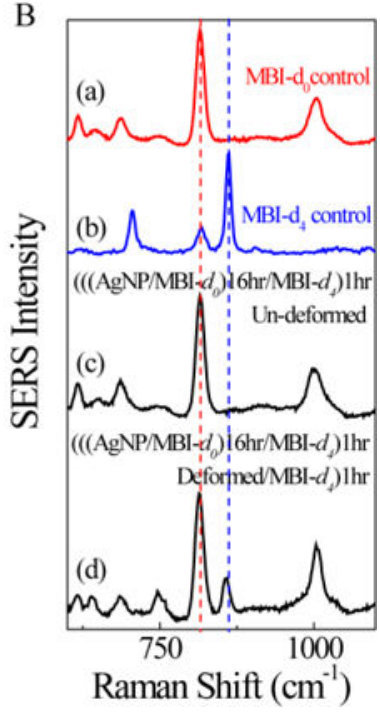

Figure 5.

(A) Schematic representation of the SERS spectral acquisition process. (B) Representative SERS spectra of (a) MBI- $d_{0}$ and (b) MBI- $d_{4}$ adsorbed onto colloidal AgNPs, (c) ((AgNP/ MBI- $\left.\left.d_{0}\right) 16 \mathrm{hr} / \mathrm{MBI}-d_{4}\right) 1 \mathrm{hr}$ before deformation and, (d) (((AgNP/MBI- $\left.\left.d_{0}\right) 16 \mathrm{hr} / \mathrm{MBI}-d_{4}\right) 1 \mathrm{hr} /$ deformed/MBI- $\left.d_{4}\right) 1 \mathrm{hr}$. The initial concentrations of MBI- $d_{O}$ and MBI- $d_{4}$ in all the samples are $1 \mathrm{mM}$. For the sequential sample, more than monolayer of MBI- $d_{O}$ is first mixed with AgNPs to allow complete ligand adsorption. The peak marked with (red dotted line) and (blue dotted line) are the MBI- $d_{O}$ and $\mathrm{MBI}-d_{4}$ features, respectively. The spectral integration time was $20 \mathrm{~s}$ with a $632.8 \mathrm{~nm}$ laser and the laser power on the sample was $5 \mathrm{~mW}$. Spectra were scaled and offset for clarity. Three independent measurements were acquired and averaged for each sample. 

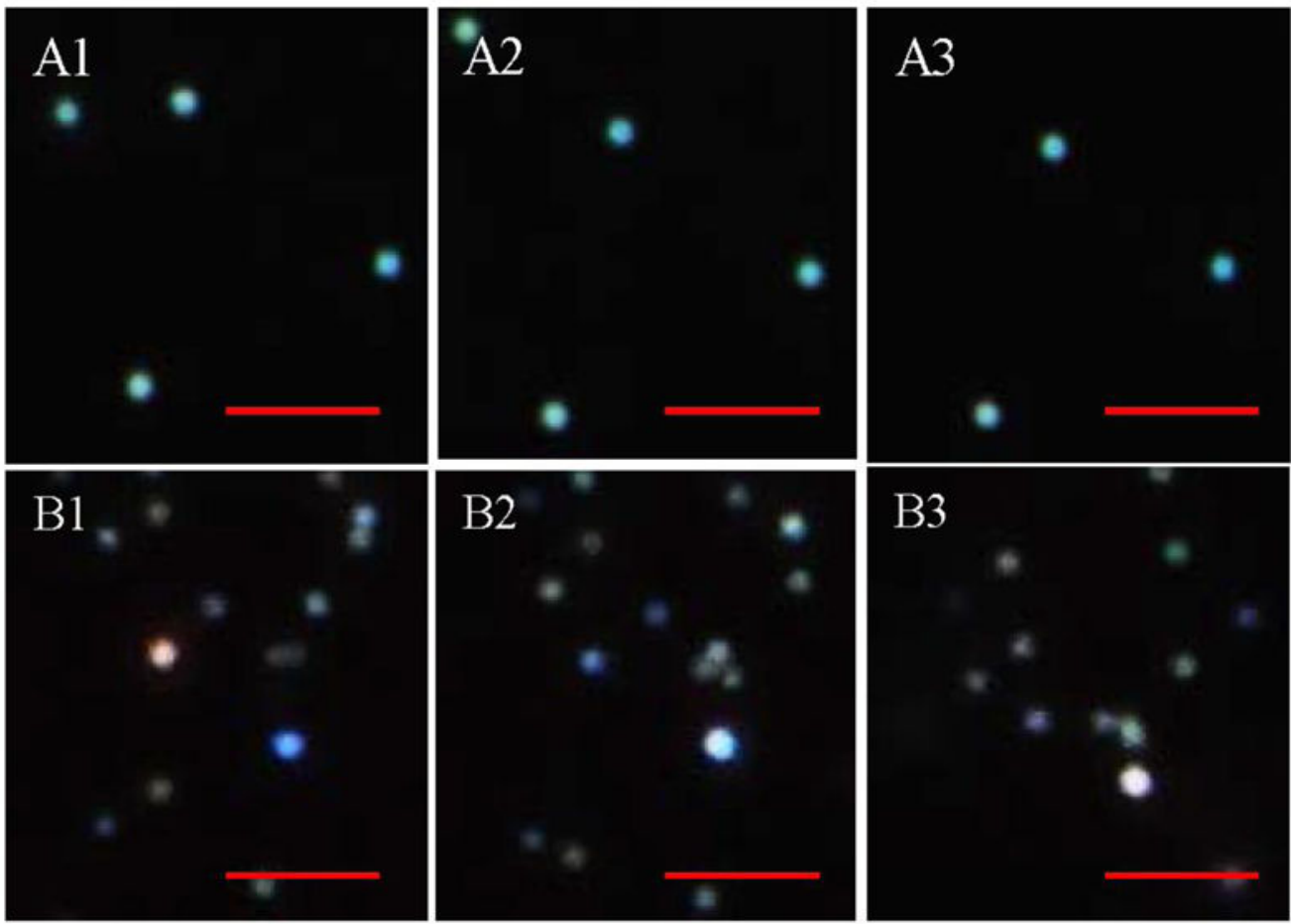

Figure 6.

Dark field images of nanoparticles in time as they translate and rotate under Brownian motion in a glycerol/water solution. A(1-3) un-deformed AgNPs translating but not showing appreciable differences in color as they rotate. $\mathrm{B}(1-3)$ deformed AgNPs blinking and changing colors as they rotate. The scale bar is $8 \mu \mathrm{m}$. 